\title{
SOME SPECTRAL PROPERTIES OF FOURTH ORDER DIFFERENTIAL OPERATOR EQUATION
}

\author{
Nigar M. Aslanova, Mamed Bayramoglu and Khalig M. Aslanov
}

Abstract. We consider boundary value problem for fourth order differential equation with unbounded discrete operator coefficient. One of the boundary conditions involves the $\lambda$ parameter. The asymptotics of spectrum of corresponding selfadjoint operator is obtained. We also calculate the trace of that operator.

Mathematics subject classification (2010): 34B05, 34G20, 34L20, 34L05, 47A05, 47A10.

Keywords and phrases: Hilbert space, differential operator equation, spectrum, eigenvalues, trace class operators, regularized trace.

\section{REFERENCES}

[1] J. WALTER, Regular eigenvalue problems with eigenvalue parameter in boundary condition, Math. Z. 133 (1973) 301-312.

[2] Charles T. Fulton, Two-point boundary value problems with eigenvalue parameter contained in the boundary conditions, Proceedings of the Royal Society of Edinburgh 77 A (1977), 293-308.

[3] V. I. GorbachuK, M. A. RYBAK, On boundary value problems for Sturm-Liouville operator equation with spectral parameter dependent boundary condition, Direct and Ill posed problems of scattering theory, Kiev, 3-13, (1981).

[4] M. A. RYBAK, On aymptotics of eigenvalue distribution of some boundary value problems for SturmLiouville operator equation, Ukr. Math. Journal 32, no. 2 (1980), 248-252 .

[5] V. I. GorbachuK, M. L. GoRBACHUK, Boundary value problems for operator, Differential equations, Kluwer, Dordrecht, 1991.

[6] F. S. Rofe-Beketov, A. M. Kholkin, Spectral analysis of differential operators, Interplay betuwun spectral and ascillatory properties. WSPC. Singapore, 2005, 46.

[7] V. A. SAdovnichil, V. E. Podols KII, Traces of operators, Russian Math. Surveys 61: 5 (2006), $885-953$.

[8] F. Gesztesy, R. Weikard, M. Zinchenko, On spectral theory for Schrödenger operators with operator valued potentials, Journal of Differential Equations, January 2013, doi: 10.1016/j.jde.2013.05:022-Source:archiv.

[9] N. M. ASLANOVA, Trace formula of one boundary value problem for operator Sturm-Liouville equation, Siberian Journal of math. vol. 49, no. 6, 1207-1215 (2008).

[10] N. M. ASlanova, Investigation of spectrum and trace formula of operator Bessel equation, Siberian Math. Journal, July, August, vol. 51, no. 4, 721-737 (2010).

[11] M. Bayramogly, N. M. Aslanova, Eigenvalue distribution and trace formula of operator SturmLiouville equation, Ukr. Math. Journal, vol. 62, no. 7, 867-877 (2010).

[12] M. Bayramogly, N. M. Aslanova, Study of the asymptotic eigenvalue distribution and trace formula of a second order operator differential equation, Boundary value problems 2012: 8, doi: 10.1186/1687-2770-2011-7, p. 22, (2012).

[13] N. M. Aslanova, The asymtotics of eigenvalues and trace formula of operator associated with one singular problem. Boundary value problems, 2012: 8, doi: 10.1186/1687-2770-2012-8, p. 12, (2012).

[14] N. M. Aslanova, M. Bayramogly, On generalized regularized trace formula of fourth order differential operator with operator coefficient, Ukr. Math. Journal, vol. 66, no. 1, 128-134, (2014). 
[15] V. I. GoRBACHUK, On aymptotics of eigenvalues of boundary-value problems for differential equations in space of vector functions, Ukr. Math. Journal, vol. 27, no. 5, 651-664, (1975).

[16] F. G. Maksudov, M. Bayramogly, A. A. AdegeZalov, On regularized trace of Sturm-Liouville operator on finite segment with unbounded operator coefficients, Dokl. AN SSSR, vol. 277, no. 4, 795-799 (1984). 